The complete genomic DNA sequence of an organism in principle yields its full coding potential, and gives the possibility of describing in a comprehensive fashion the structures and functions of proteins, and their organization into the pathways and networks that control cellular behaviour ${ }^{2}$. The vast literature on these topics seems likely to grow exponentially with the refinement of tools for rapid proteomic analysis. We feel that the value of this information, and its ability to serve as the basis for modelling of cellular responses to external signals, will depend on its organization into a readily accessible electronic format.

One approach to such a database makes use of the observation that a common theme in cellular events is the assembly of proteins into complexes, through specific modular interactions ${ }^{3}$. A growing number of such interactions use domains and recognition motifs that can be readily identified by primary sequence analysis, and are therefore predictable ${ }^{4}$. This notion can be extended to encompass the interactions of distinct types of macromolecules with one another, and with small molecules. Although not all cellular phenomena can be described in these terms, the concept provides a useful starting point from which to organize data.

The purpose of the workshop was to explore continuing efforts to design databases of protein-protein interactions, and to solicit input as to the best way forward. We considered the creation of a centralized, freely available, public submission database as an achievable and highly desirable goal for the next generation of cellular analysis. Such an undertaking will be complex and prone to numerous pitfalls, but we believe it is an inevitable evolution of current biological databases. Furthermore, we consider it essential if we are to understand more fully how cellular function is controlled.

A transcript of the workshop will be posted on the NIGMS website. As this initiative proceeds, we will solicit broader input from the scientific community.

Marvin Cassman*, Tony Hunter $\dagger$,

Tony Pawson $\ddagger$

*National Institute of General Medical Sciences,

National Institutes of Health, Bldg 45, 2AN12B,

Bethesda, Maryland 20892, USA.

$\dagger$ Salk Institute, 10010 North Torrey Pines, La Jolla, California 92037, USA

$\ddagger$ Samuel Lunenfeld Research Institute, Mount Sinai Hospital, 600 University Avenue, Toronto, Ontario M5G 1X5, Canada

1. Abbott, A. Nature 402, 219-220 (1999).

2. Plowman, G. D., Sudarsanam, S., Bingham, J., Whyte, D

\& Hunter, T. Proc. Natl Acad. Sci. USA 96, 13603-13610 (1999)

3. Pawson, T. \& Scott, J. D. Science $278,2075-2080$

(1997).

4. Songyang, Z. et al. Cell 72, 767-778 (1993).

\section{Debating controversies can enhance creativity}

Sir- I could not agree more with the author of your Opinion "Resolution to enhance confident creativity" (Nature 403, $1 ; 2000)$ that "there will always be established and influential scientists who ... are resistant to looking beyond their long-held scientific assumptions". I also agree that "too many of today's creative scientists lack long-term security" and that "good but unconventional ideas are probably falling by the wayside".

Peer review is important for ensuring the quality of published work and proposed studies. This quality check can prevent false information being disseminated and funds being wasted. But peer review can also restrict creativity. What can be done to improve the system?

With the emergence of electronic publications, which do not have to rely on a fixed format, the reviewing/citation components can now be integrated into publications. Supplemental information and hyperlinks can be added to electronically published papers to connect them with related information - a review or a follow-up research article, say. In this way, the value of the published work is automatically revealed through the reading of linked literature. Ultimately, prepublication review might even be eliminated and replaced with continuous post-publication review, creating a free atmosphere for expressing creative ideas.

I have started such an experiment and created an electronic journal, Logical Biology (http://logibio.com), dedicated to debating controversial issues and promoting logic as a tool for scrutinizing long-held conventional views in biology: for example, the nature of bacterial life. Some people may dismiss this type of publication, but, in the interest of fostering creativity, isn't it worth a try? The primary goal of scientific publication is, after all, not validation but communication.

Shi V. Liu

Department of Microbiology and Immunology, School of Medicine, MCP Hahnemann University, 2900 Queen Lane, Philadelphia, Pennsylvania 19129, USA

ShiVLiu@logibio.com

\section{Time for an aspirin}

Sir-I found it painfully hard to read the first of the "Timescales" pages in the Impacts of Foreseeable Science supplement (Nature 402 (suppl.), C17; 1999). The vaguely coloured and seemingly meaningless background combined with the rather small compressed and low-contrast typeface gave me a headache. It's ugly, besides.

Artists should be on tap, not on top. Marvin Minsky

MIT Media Laboratory, 20 Ames Street,

Cambridge, Massachusetts 02139, USA

\section{The myth of well-funded German research}

Sir-You reported on difficulties in financing genome research in Germany (Nature 402, 706; 1999). Two pages later, we read: "Lots more cash for UK universities". These articles are symptomatic of the current funding crisis in German research. Because of the country's past successes and its relative wealth, it is not generally known that research in Germany is no longer well funded. None of the Länder (states) provides enough funds to replace equipment in university laboratories - my institute has averaged less than 1 per cent of equipment costs per year for the last 20 years. Even before reunification in 1990 placed serious pressure on public funding, increases in federal support for the Deutsche Forschungsgemeinschaft (DFG) were very small.

In my field, biomedicine, research funding trails that of competing countries. In the United Kingdom, support from all sources in 2000 amounts to at least US\$24 per head of population, compared with $\$ 15$ in Germany. In the United States, biomedical funding from the National Institutes of Health (NIH) and Howard Hughes Foundation alone represents \$74 per head of population — five times higher than all such funding in Germany. Japan intends to double its science budget and the NIH has started very well on its target of doubling US biomedical research funds within five years. In the United Kingdom, funding by the private Wellcome Trust alone exceeds all equivalent funding from the DFG. Although the DFG has given out 59 per cent more for standard grants over the past ten years, this is no increase at all when corrected for inflation and increases in population.

Germany has few natural resources and relies on its best minds to produce knowledge and wealth. It needs a huge increase in public spending on research and a massive infrastructure-replacement fund from federal and state governments. It is time for scientists and learned societies to begin lobbying much more intensively: German science will only be able to compete if funding is tripled or quadrupled in the next five years. Geoffrey A. Manley Institut für Zoologie, Technische Universität München, Garching, Germany 